\title{
Jiti Gao: Nonlinear time series - semiparametric and nonparametric methods
}

\author{
Chapman \& Hall/CRC, xii + 237 pp., US \$79,95, GB $£ 42,74$, \\ $€$ 90,39. ISBN 1-58488-613-7
}

\section{Matthias Fischer}

In the last two decades, there has been growing interest in the theory and applications of nonlinear time series models. Whereas the main focus originally was on parametric and nonparametric modelling, semiparametric methods have recently found their way into time series literature. With his book Jiti Gao provides a comprehensive overview of such semiparametric time series modelling and recent developments therein. It is primarily addressed to postgraduate students and to researchers who are interested in gaining deeper insight into nonlinear time series using semiparametric methods. The merits of Gao's book lie in the presentation of past and current theoretical research results on that topic (as a closer look at the referenced literature also reveals), plus some empirical application from climatology and finance. The contents can be briefly summarized as follows: Chap. 1 is mainly dedicated to some preliminaries, examples as well as simple time series models. Semiparametric estimation and specification methods are presented in Sects. 2 and 3. Whereas Chap. 2 is concerned with the study of estimation problems of various parameters and functions involved in semiparametric models, Chap. 3 mainly discusses semiparametric specification of various conditional moments. Chapter 4 deals with semiparametric model selection with special emphasis on cross validation and penalty function methods. The main focus of Chap. 5 lies on semiparametric estimation and specification of continuous-time models. Chapter 6 connects long range dependence to semiparamtetric methods and discusses some very recent theoretical and empirical results. Finally, Chap. 7 is presents to some technical lemmas and an excursion on asymptotic normality and expansions.

To summarize, this book covers many recent developments of modern semi-parametric time series analysis. I strongly recommend it to anybody who wants to gain comprehensive insight into this subject on an advanced level.

\footnotetext{
M. Fischer $(\varangle)$

Universität Erlangen-Nürnberg, Nuremberg, Germany

e-mail: domafisch@freenet.de
} 